\title{
Performance of LoRa for Bike-Sharing Systems
}

\author{
Daniele Croce, Domenico Garlisi, Fabrizio Giuliano, Alice Lo Valvo, Stefano Mangione, Ilenia Tinnirello \\ Department of Engineering, Università di Palermo, Italy \\ Corresponding author: Domenico Garlisi, domenico.garlisi@unipa.it
}

\begin{abstract}
Today bike sharing systems are becoming popular in many cities as short-distance transit vehicles. More than $\mathbf{1 8}$ million bicycles are available worldwide for public use and one of the main problems that afflicts such sharing systems is the loss of bikes, which can be stolen or simply left in unknown locations. Thus, many bikes are docked or tracked using GPS and costly cellular connections.

In this paper, we consider the emerging Long Range (LoRa) technology for use in bike sharing systems. LoRa exploits free ISM bands and has been conceived for low power and low data rate applications. Additionally, LoRa is characterized by large cells and heterogeneous application domains, which may lead to extremely high numbers of devices coexisting in the same cell. Thus, in this paper we study the scalability limits of a typical LoRa cell, showing that the performance of LoRa in crowded scenarios can be quite limited when using high spreading factors (SFs). Then, we design and realize a prototype of LoRa tracker module that can be embedded in a bike and we test its use in a large area of Palermo city, Italy. According to our knowledge, this is the first paper that study the performance of LoRa for bike sharing systems.
\end{abstract}

Index Terms-LoRa, LoRaWAN, Low Power Wide Area Networks (LPWAN), Automotive, Vehicular Network (VANET), bike, bike-sharing, tracker, IoT.

\section{INTRODUCTION}

Based on a recent report from the World Health Organization, air pollution in many developing countries has become the greatest threat to human health, and is responsible for one in eight deaths each year. Indeed, the air in the world big cities contains high levels of dangerous particulate that contributes to an estimated 7 million premature deaths each year [1].

Several public administrations are promoting the usage of bike in order to reduce the air pollution issue, since bicycles do not use any fuel. While cars significantly pollute the air over the course of their lifespan, using bikes is a powerful way to reduce fuel-based pollution caused by vehicles. In many cities, bikes are now used as short-distance transit vehicles and bike sharing systems are becoming popular in many metropolitan areas worldwide. Indeed, bike sharing systems have the following advantages: i) more effective usage of pubic transportation assets and resources; ii) reduced number of fuel vehicles which in turn reduces the carbon emission, traffic congestion, and noise; and iii) improved health of the citizens.

Bike-sharing systems have come a long way since the first system launched in Amsterdam in 1965: as of 2013, there were 535 bike sharing services worldwide, in 49 countries, with a total fleet of over 500000 bicycles, and as of May 2018 , more than 1,600 bike-sharing programs were in operation worldwide, providing more than 18 million bicycles for public use ${ }^{1}$. Today e-bikes are also becoming very popular, exploiting high performance control techniques for electrical drives where speed and torque are suitably controlled [2], [3], [4], [5].

One of the problems that afflicts bike sharing systems is the loss of bikes, which can be stolen or simply left in unknown locations. Bike docking systems work reasonably well, but these systems require that users get and return their bikes at defined locations. More recently, due to the popularity of smartphones and cashless mobile payments, the constraint of fixed docking stations in traditional bike-sharing service has been removed: bikes use GPS and cellular connections to send positioning coordinates to the network which can then identify and locate locked bikes. This data can be used to collect information about transportation routes and user riding profiles as well as offering additional services, such as enroute business promotions or pick-up and delivery [6], [7], [8], [9]. Finally, new dockless bike sharing systems also bring complex management problems such as how to reallocate the bikes after a whole day operation [10], [11], [12].

Recently, the Ofo bike sharing company announced to include in their systems a new GPS tracker based on the Long Range (LoRa) transmission technology [13]. LoRaWAN is a new Low Power Wide Area Network (LPWAN) network exploiting the LoRa transmission technology for enabling power efficient wireless communications over very long distances. Differently from NB-IoT [15], which is based on LTE cellular networks, LoRAWAN works in unlicensed bands, without a SIM and with very limited investments on network infrastructures. These features make this technology very suitable for connecting simple objects even in rural areas. LoRaWAN networks are based on a star topology: every node can reach directly one (or more) Internet connected sink nodes called gateways. However, given the fact that gateways cover a wide area, a large number of nodes have to share the communication medium. For this reason, LoRa provides different possibilities to orthogonalize transmissions as much as possible - Carrier Frequency (CF), Spreading Factor (SF), Bandwidth (BW), Coding Rate (CR) - and provide simultaneous collision free communications. Despite of the robustness of the LoRa PHY [14], patented by Semtech, in metropolitan scenarios where multiple gateways can be installed, the scalability of this technology is still under investigation [16]. Nevertheless, LoRa can be used to economize over standard cellular technologies and allows to reach higher distances compared to WiFi or

\footnotetext{
${ }^{1}$ https://civitas.eu/content/list-bicycle-charing-systems
} 
ZigBee, which are known to suffer from interference problems [17], [18], [19].

In this paper, we study the performance of LoRa for bike sharing systems. First, we study in simulation the scalability limits of a typical LoRa cell, showing that the performance of LoRa in crowded scenarios can be quite limited when using high spreading factors (SFs) and that fading has a moderate impact on the cell capacity. Then, we design and realize a prototype of LoRa tracker module that can be embedded in a bike and we test its use in a large area of Palermo, Italy. From our literature research, this is the first paper that presents a complete system architecture for bike sharing that uses LoRaWAN technologies.

The rest of the paper is organized as follows. Section II presents the main works in the current literature and background on the LoRa technology is described in Section III. In Section IV we present the simulation results, we also analyze some scalability issues of LoRa when the number of bikes increases. Section V presents the architecture design of the LoRa tracker module and in Section VI we report on the coverage experiments in the city of Palermo, Italy. Finally, Section VII presents conclusions and future work.

\section{RELATED WORK}

Many of the current studies on bike sharing systems analyze the bike redistribution problem after daily operations. For example, in [10], the authors attempt to infer bike trip patterns directly from station status data. However, the station status data do not contain information about where the bikes come from and go to, this implies possibles errors in the evaluation of the bike trips. To solve this problem, the authors conduct an empirical study on a sample bike trip dataset to gain insights about the structure of bike trips. They then formulate the trip inference problem as an ill posed inverse problem, and propose a regularization technique to incorporate the apriori information about bike trips to solve the problem. In [11] the bike redistribution problem is compared with the empty container reposition problem. The authors propose a simulation study on the effects of the rental information and bike redistribution strategies to the bike sharing systems. In particular, four simulation models are developed to analyze the impacts of different redistribution strategies with different levels of bike rental information. In [12], the Authors use the Big Data analytics to produce an integrated method for reallocating dockless bikes. They collect and preprocess the GPS data of dockless bikes and cluster these bikes into several groups, examining large data sets to uncover hidden patterns, unknown correlations, market trends, customer preferences and other useful business information. Then, the authors formulate the reallocation of bikes using an integer programming model.

These works related to the bike reallocation problem do not consider how the bike data is recovered, and which is the best solution for bike tracking. Indeed, in [10], [11], [12] is clear the need to track the bike but the technology used to gather this information is not presented or, in the case of [10], the authors consider a dataset with only the docking stations position.
Other than for bike tracking, the use of LoRa technology as tracking system is presented in [20] for IoT environments. In this case, LoRa is used to develop a secure solution for tracking different objects indoors and outdoors having unique IDs. The system presents different requirements for privacy and accuracy of the location information. The designed prototype uses LoRa communication to transmit location data in support of an anti-theft and protection system based on device tracking. However, the authors do not take into consideration the coverage and scalability limits of the LoRa technology. In [21], [22], the authors study the impact of imperfectorthogonality in LoRa spreading factors (SFs) in simulation and real-world experiments. They show that collisions between packets of different SFs can indeed cause packet loss if the interference power received is strong enough. The paper results show that non-orthogonality of the SFs can deteriorate significantly the performance especially of higher SFs (10 to 12). The results demonstrate that inter-SF collisions are indeed an issue in LoRa networks and, thus, allocating higher SFs to the end device far from the gateway not necessarily improve their link capacity, in case of congested networks. However, [21], [22] do not study coverage issues in real metropolitan areas.

Finally, [23] presents a LoRa cell traffic generator, able to emulate the behavior of thousands of end device deployed in the same cell by using a single Software Defined Radio (SDR) platform. Differently from traditional generators, whose goal is creating packet flows which emulate specific applications and protocols, the proposed LoRa cell traffic generator combines the radio signals as seen by a gateway, given by the super-position of the signals transmitted by multiple sensors simultaneously active on the same channel. However, coverage problems typical of the urban canyons are not considered in this study.

\section{LORAWAN TECHNOLOGY}

In this section we briefly describe the LoRaWAN network architecture and MAC/PHY protocols, in order to present the main features that affect the network capacity.

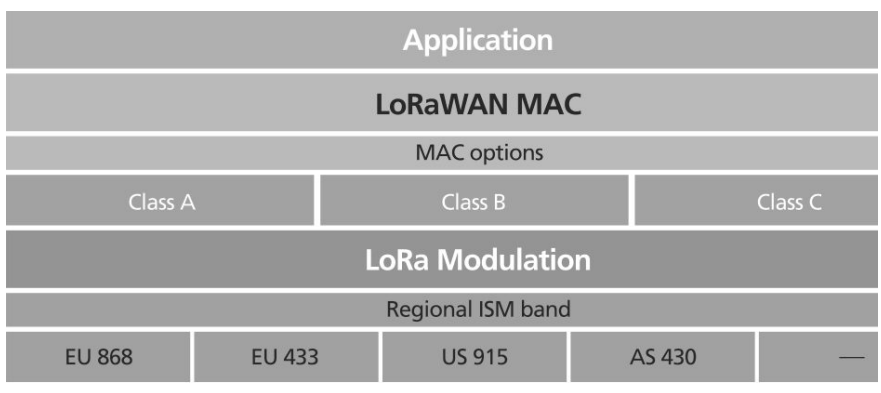

Fig. 1. LoRa technology stack.

As shown in Fig. 1, LoRaWAN is a protocol specification built on top of the LoRa technology that uses ISM bands (868Mhz with 3 default channels in Europe) with a limited 


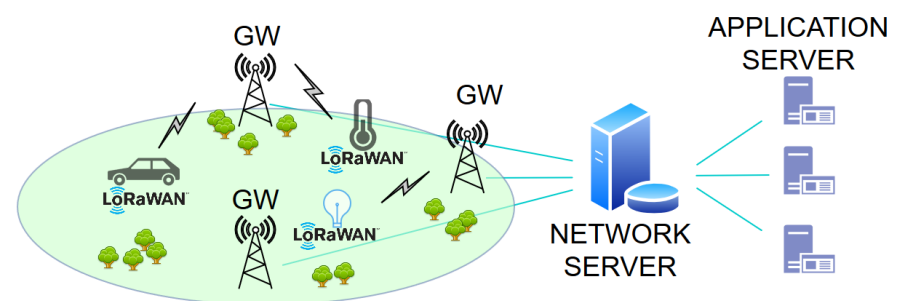

Fig. 2. LoRa network architecture.

duty cycle allowed to each device [13]. The typical LoRaWAN network consists of end devices (sensors or actuators, in our cases the bicycles), multiple gateways which forward packets to a network server and to the application, as shown in Figure 2. A typical LoRaWAN network topology is a "star of stars", which means that groups of end devices are connected to gateways via LoRa wireless links while the gateways are connected to a remote Network Server via more standard technology, like WiFi, 3G, LTE or IP network. LoRaWAN also proposes an Adaptive Data Rate (ADR) mechanism in order to adapt the transmission characteristics (data rate, spreading factor, and bandwidth) to the propagation conditions. Data rates range from DR0 (the lowest data rate) to DR7 (the highest data rate) roughly corresponding to the SFs 7 to 12 . There can be multiple gateways in a LoRa deployment, and the same data packet can be received (and forwarded) by more than one gateway.

LoRaWAN networks are advertised to be able to handle millions of devices. Moreover, the network server will be responsible for the optimization of all of these nodes. Even if the data rate in sensor networks is significantly lower than in traditional networks, the network operators should evaluate the scaling issue of the network. Different from traditional cellular networks, the end-devices are not associated with a particular gateway in order to have access to the network. The gateways serve simply as a link layer relay and forward the packet received from the end-devices to the network server after adding information regarding the reception quality. Thus, an end-device is associated with a network server, which is responsible for detecting duplicate packets, choosing the appropriate gateway for sending a reply (if any), consequently for sending back packets to the end-devices. Logically, gateways are transparent to the end-devices. LoRaWAN specifies the behavior of the devices, but not the behavior of the network server. For this reason it is important to maintain the load on the network low, and the network server has to enforce this by sending MAC commands to the devices. However, the correct behavior of the network server is not easy to be evaluated [16].

Gateways are usually equipped with multiple transceivers for receiving simultaneously on multiple (configurable) frequency channels, while end devices can dynamically select one transmission channel, among a set of available ones, at each transmission attempt. LoRaWAN provides an open source MAC layer that is based on a simple Aloha protocol, in order
TABLE I

SIMULATION PARAMETERS.

\begin{tabular}{ll}
\hline Parameter & Value \\
\hline Carrier Frequency & $860.0 \mathrm{MHz}$ \\
Transmission Power & $14 \mathrm{dBm}$ \\
Bandwidth & $500 \mathrm{kHz}$ \\
Code Rate (CR) & $4 / 5$ \\
Message size & $20 \mathrm{Bytes}$ \\
Message Period & $1 \mathrm{pkt} / 90 \mathrm{sec}$ \\
Number of gateway & 1 \\
Number of nodes & {$[50-2000]$} \\
Path loss attenuation exponent & 4 \\
Fading variance & {$[0-10] \mathrm{dB}$} \\
\hline
\end{tabular}

to minimize the complexity and the energy consumption of the devices. End devices do not perform carrier sense and can be set with three different class of work (named A, B and C) according to the receiver window.

\section{Scalability Results}

In order to verify the scalability of the LoRa network, we used the simulator in [21] and defined a single cell scenario with two different radius values, $12 \mathrm{~km}$ and $34 \mathrm{~km}$. In the first case it is possible to use any SF value for any node, because all nodes are inside the cell coverage of the lowest SF7. All the simulation parameters are reported in table I.

For comparison purposes, we refer to the same metric used in [21] for characterizing the performance of LoRa networks, namely the Data Extraction Rate (DER) defined as the quota of packets correctly received by the gateway. The achievable DER depends on the position, number and rate of LoRa end devices, as well as on allocation of SFs among the end devices.

In the simulations we considered a log-normal fading model of increasing $\sigma=0,3,6,10 \mathrm{~dB}$. Fig. 3 shows the performance of SFs 7, 9 and 12 under fading, in presence of capture effects. Interestingly, while fading affects lower SFs (7 to 9), the DER of the higher SF 12 remains virtually unchanged even in presence of strong fading. This is due to the fact that faded packets do not compete at the receiver and thus the probability of collision is reduced, leaving the other packets more chances to be received and compensating the risk of being lost because of fading. Similar results can be obtained using other SFs, which we omit for sake of simplicity. From the shown results, it is clear that the capacity of LoRa is quite limited when using high SFs and thus, allocating them to achieve longer distances could not necessarily improve the link capacity in case of congested networks. Indeed, higher SFs could be more prone to collisions due to longer transmission times. On the other hand, using low SFs might reduce the cell coverage especially in urban canyons.

\section{LORA TRACKER MODULE}

To test the performance of LoRa in urban settings, we developed a prototype of LoRa tracker module composed 

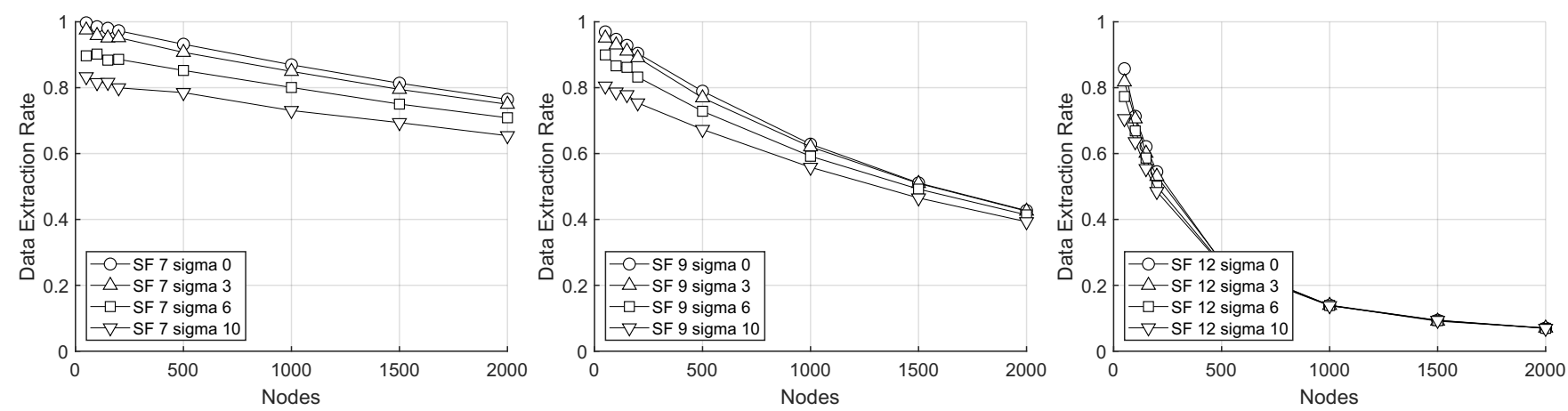

Fig. 3. Performance of LoRa in presence of log-normal fading $(\sigma=0,3,6,10 \mathrm{~dB})$ when Spreading Factor is 7 (left), 9 (center) and 12 (right).

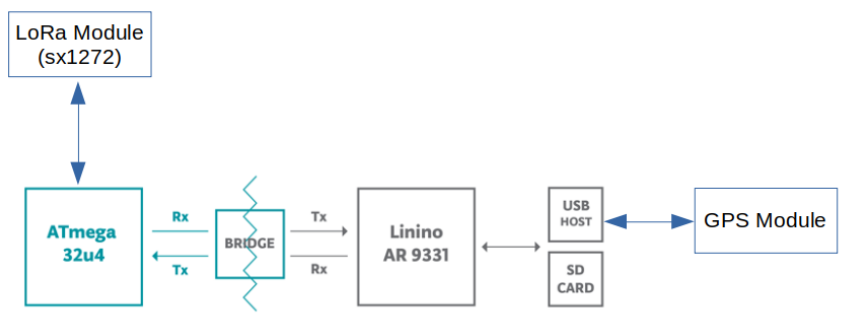

Fig. 4. LoRa Device Architecture.

of three elements: 1) an Arduino Yun Board, 2) a LoRa transmission module (based on Semtech chip sx1272) and 3) a USB GPS module. The architecture of the device is shown in figure 4. The Arduino Yun includes both Linux and Arduino environments, while the core of the LoRa tracker module is represented by Semtech SX1272 chip. This chip, uses the SPI interface to give access to its registers. To manage the LoRa module, a sketch has been implemented and run on Arduino environment which has an ATmega32U4 microcontroller also equipped with an SPI interface. The used USB GPS module is a Columbus V-800.

The GPS data is acquired by the Linux environment of the Arduino Yun, which exchanges information with the Arduino ATmega microcontroller. The Arduino sketch then implements the communication primitives with the LoRa Module and triggers the data transmission of the GPS coordinates towards the gateway.

From the hardware perspective, Arduino Yun and SX1272 have two different working voltage (5V and $3.3 \mathrm{~V}$ respectively). For this reason, we designed and developed an adaptation circuit that adapts the two different voltages (interface shield). In figure 5 is showed the final integration of the tracker module. For the sketch implementation, the libraries provided by Libelium were used which include the network architecture and a packet format.

\section{EXPERIMENT RESULTS}

In order to test the designed system and analyze the effect of coverage, we performed a measurement campaign in an area of about 50 square $\mathrm{Km}$ of the Palermo city, comprising both

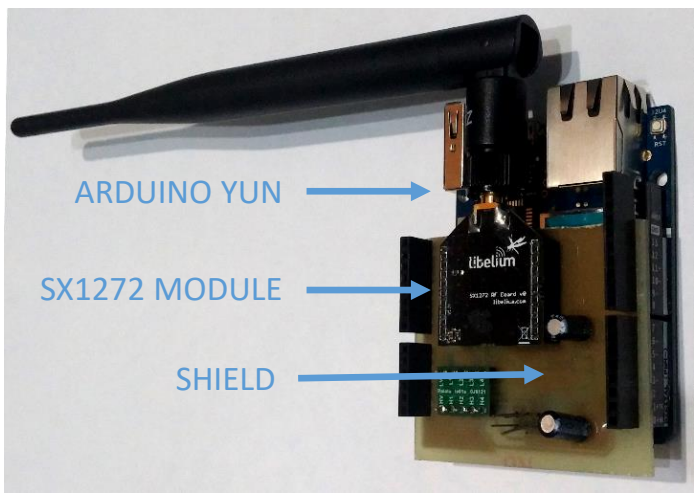

Fig. 5. Arduino Adaptation Board.

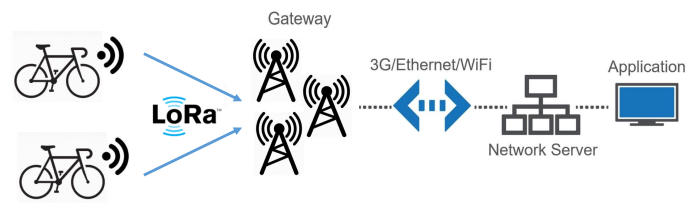

Fig. 6. LoRaWAN network architecture in our application scenario.

urban and sub-urban settings. Figure 6 shows the architecture of the developed system. The LoRa gateway (a commercial RAK7249 Multibandwidth LoRaWAN GW working in $868 \mathrm{MHz}$ Bandwidth) was installed at the top of a campus building as shown in Figure 7. The gateway is connected via Ethernet connection to internet, the received LoRa packets are forwarded to our custom network server. Figure 8 shows the position of the LoRa gateway in the explored area as well as the maximum distance reached. We tested the proposed tracker module at different transmission distances by setting the output power level to $14 \mathrm{dBm}$ and with conservative transmission parameters of $\mathrm{BW} 125 \mathrm{kHz}$ and $\mathrm{SF} 12$. To obtain a precise coverage mapping, the module sends the GPS coordinates with a transmission period of 3 seconds, while the center frequency is $868.1 \mathrm{MHz}$. In figure 8 the pin color represents the RSSI of the packet: red is used for packets received between -130 , and $-120 \mathrm{dBm}$, yellow for the range -120 to $-94 \mathrm{dBm}$ and green for the range -93 to $-82 \mathrm{dBm}$ and finally blue for the range 


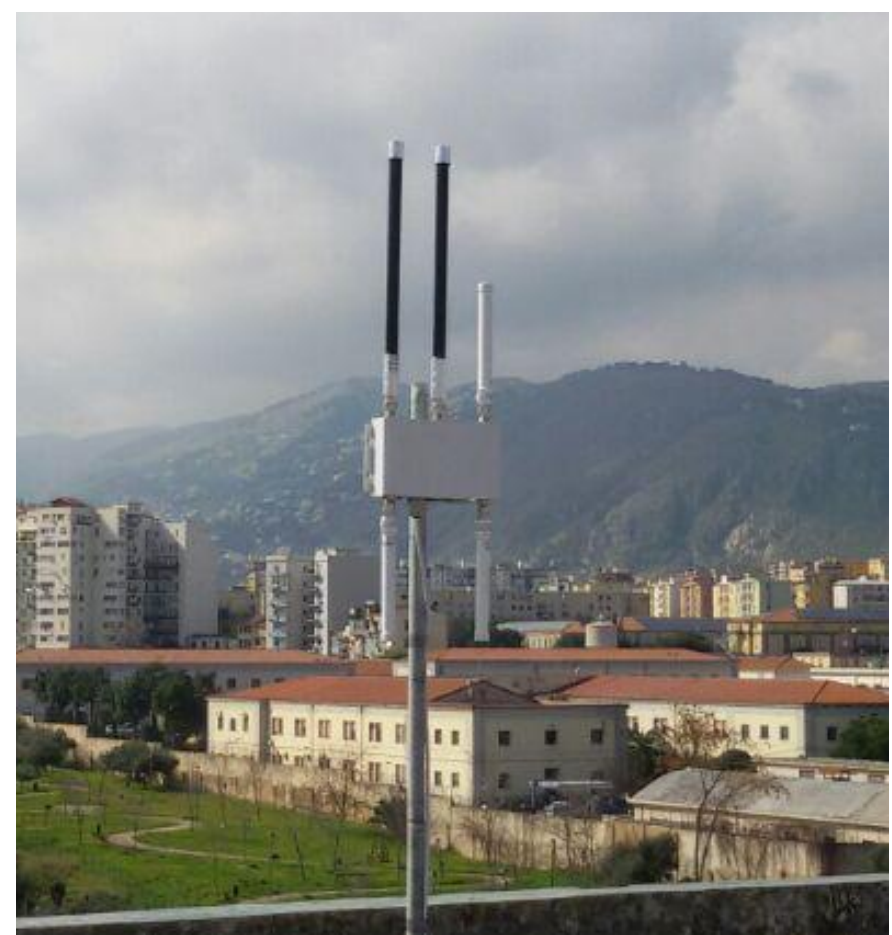

Fig. 7. LoRa Gateway installation.

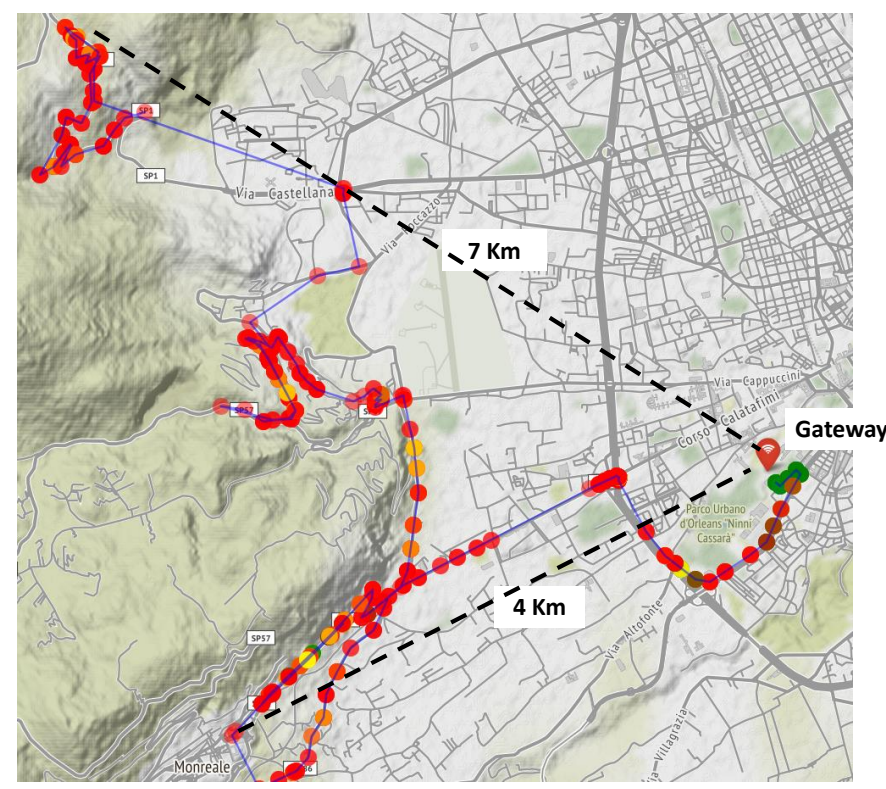

Fig. 8. Large Experiment coverage Maximum distance achieved $7.3 \mathrm{~km}$

between -81 to $-69 \mathrm{dBm}$. Finally, figure 9 shows the RSSI values distribution of the received frames.

These experimental results show that the coverage is very limited in the urban canyons, with "holes" in non line of sight scenarios. On the other hand, long distances, almost $8 \mathrm{~km}$, can be reached when the gateway is in direct visibility of the end device. Thus, positioning of the gateway is critical and should be carefully studied for an optimal coverage, e.g. placing the

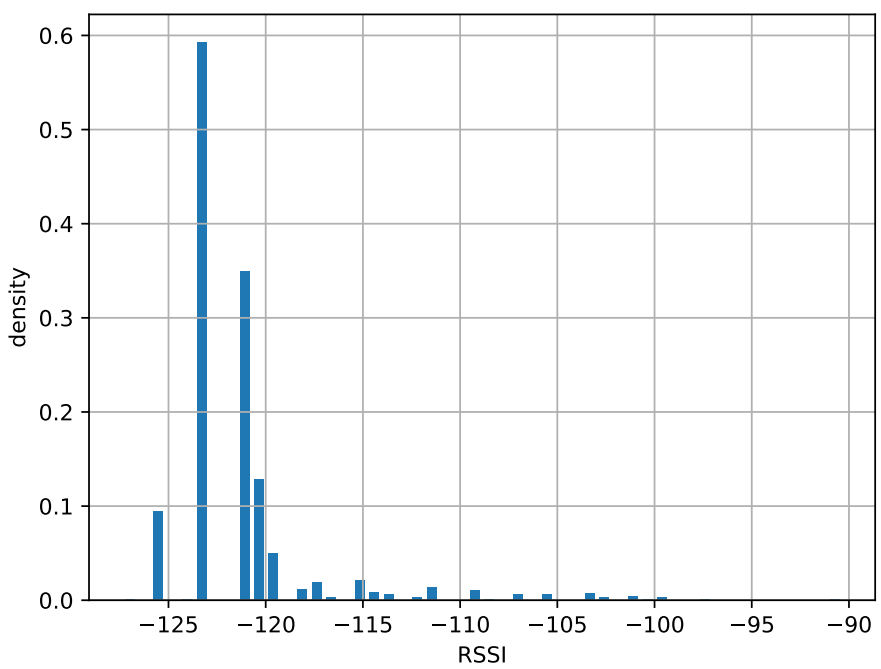

Fig. 9. RSSI experiment distribution

gateways on high mountains, buildings or towers.

\section{CONCLUSIONS}

In this paper we have explored the use of the emerging LoRa technology for bike sharing systems. Today bike sharing systems are becoming popular in many cities as short-distance transit vehicles and many bikes are tracked using GPS and costly cellular connections. Instead, LoRa exploits the free ISM bands and has been conceived for low power, low data rate applications. However, LoRa is characterized by large cells and heterogeneous application domains, which may lead to extremely high numbers of devices coexisting in the same cell.

Thus, in this paper, we studied in simulation the scalability limits of a typical LoRa cell, showing that the performance of LoRa in crowded scenarios can be quite limited when using high SFs and that fading has a moderate impact on the cell capacity. Moreover, we realized and tested a prototype of LoRa tracker that can be embedded in a bike and we test its use in a large area of Palermo, Italy. Our results show that the capacity of LoRa is quite limited when using high SFs and that the cell coverage is critical in urban canyons, while long distances can be reached in line of sight settings. Thus, positioning of the gateway is critical and should be carefully studied for an optimal coverage in urban environments.

\section{ACKNOWLEDGEMENTS}

This research was partially supported by the Interreg V-A Italia-Malta (2014-20) project "I-Access", (DDG 259/SV DRP 31.5.18).

\section{REFERENCES}

[1] World Health Organization, "7 Million Premature Deaths Annually Linked to Air Pollution," Air Quality Climate Change, vol. 22, no. 1, Mar. 2014, pp. 53-59.

[2] A. Accetta, F. Alonge, M. Cirrincione, F. D'Ippolito, M. Pucci, R. Rabbeni, and A. Sferlazza, "Robust control for high performance induction motor drives based on partial state-feedback linearization," IEEE Transactions on Industry Applications, vol. 55, no. 1, pp. 490-503, 2019. 
[3] F. Alonge, M. Cirrincione, M. Pucci, and A. Sferlazza, "A nonlinear observer for rotor flux estimation of induction motor considering the estimated magnetization characteristic," IEEE Transactions on Industry Applications, vol. 53, no. 6, pp. 5952-5965, 2017.

[4] F. Alonge, M. Cirrincione, F. D'Ippolito, M. Pucci, and A. Sferlazza, "Active disturbance rejection control of linear induction motor," IEEE Transactions on Industry Applications, vol. 53, no. 5, pp. 4460-4471, 2017.

[5] A. Accetta, F. Alonge, M. Cirrincione, M. Pucci, and A. Sferlazza, "Feedback linearizing control of induction motor considering magnetic saturation effects," IEEE Transactions on Industry Applications, vol. 52, no. 6, pp. 4843-4854, 2016

[6] M. A. Giambra et al., "Graphene Field-Effect Transistors Employing Different Thin Oxide Films: A Comparative Study," ACS Omega, vol. 4, no. 1, pp. 2256-2260, Jan. 2019.

[7] A. Benfante et al., "Employing Microwave Graphene Field Effect Transistors for Infrared Radiation Detection,” IEEE Photonics J., vol. 10, no. 2, pp. 1943-0655, 2018.

[8] R. Scaffaro, A. Maio, G. Lo Re, A. Parisi, and A. Busacca, "Advanced piezoresistive sensor achieved by amphiphilic nanointerfaces of graphene oxide and biodegradable polymer blends," Compos. Sci. Technol., vol. 156, pp. 166-176, 2018.

[9] P. Cusumano, "Study of voltage decrease in organic light emitting diodes during the initial stage of lifetime", Solid-State Electronics, Volume 116, (2016) Pages 30-32

[10] L. Chen and J. Jakubowicz, Inferring bike trip patterns from bike sharing system open data, 2015 IEEE International Conference on Big Data (Big Data), Santa Clara, CA, 2015, pp. 2898-2900.

[11] I. Wang and C. Wang, Analyzing Bike Repositioning Strategies Based on Simulations for Public Bike Sharing Systems: Simulating Bike Repositioning Strategies for Bike Sharing Systems, 2013 Second IIAI International Conference on Advanced Applied Informatics, Los Alamitos, CA, 2013, pp. 306-311

[12] M. Liu and X. Xu, Dockless Bike-Sharing Reallocation Based on Data Analysis: Solving Complex Problem with Simple Method, 2018 IEEE Third International Conference on Data Science in Cyberspace (DSC), Guangzhou, 2018, pp. 445-450.
[13] N. Sornin, M. Luis, T. Eirich, T. Kramp, and O. Hersent, Lorawan specification v1.0, Technical report, LoRa Alliance, 2015.

[14] Semtech. LoRa Modulation Basics. AN1200.22, Revision 2. May 2015. www.semtech.com

[15] 3GPP, "Evolved Universal Terrestrial Radio Access (E-UTRA); Physicalchannels and modulation (Release 13)," 3rd Generation PartnershipProject (3GPP), TS 36.211, Jul. 2016. [Online]. Available: http://www.3gpp.org/ftp/Specs/html-info/36211.htm

[16] M. C. Bor, U. Roedig, T. Voigt, and J. M. Alonso, Do LoRa Low-Power Wide-Area Networks Scale? In Proc. of MSWiM 2016. ACM, New York, pp. 59-67.

[17] Croce D., Garlisi D., Giuliano F., Tinnirello I. "Learning from errors: Detecting ZigBee interference in WiFi networks" 2014 13th Annual Mediterranean Ad Hoc Networking Workshop, MED-HOC-NET 2014.

[18] Tinnirello I., Croce D., Galioto N., Garlisi D., Giuliano F. "CrossTechnology WiFi/ZigBee Communications: Dealing with Channel Insertions and Deletions" IEEE Communications Letters 2016.

[19] Valck P.D., Moerman I., Croce D., Giuliano F., Tinnirello I., Garlisi D., Poorter E.D., Jooris B. "Exploiting programmable architectures for WiFi/ZigBee inter-technology cooperation" Eurasip Journal on Wireless Communications and Networking", 2014.

[20] C. Schmitt, J. Meier, M. Diez and B. Stiller, "OTIoT - A browserbased object tracking solution for the Internet of Things," 2018 IEEE 4th World Forum on Internet of Things (WF-IoT), Singapore, 2018, pp. 445-451.

[21] Croce D., Gucciardo M., Tinnirello I., Garlisi D., Mangione S. (2017) Impact of Spreading Factor Imperfect Orthogonality in LoRa Communications. In TIWDC 2017. Communications in Computer and Information Science, vol 766, pp. 165-179. Springer, Cham.

[22] D. Croce, M. Gucciardo, S. Mangione, G. Santaromita and I. Tinnirello, Impact of LoRa Imperfect Orthogonality: Analysis of Link-Level Performance IEEE Communications Letters, vol. 22, no. 4, pp. 796-799, April 2018.

[23] Michele Gucciardo, Ilenia Tinnirello, and Domenico Garlisi. 2017. Demo: A Cell-level Traffic Generator for LoRa Networks. In Proceedings of the 23rd Annual International Conference on Mobile Computing and Networking (MobiCom '17). 